\title{
Medical Image Segmentation Based on Wavelet Analysis and Gradient Vector Flow
}

\author{
Ji Zhao, Lina Zhang, Minmin Yin \\ Department of Software Engineering, University of Science and Technology Liaoning, Anshan, China \\ Email: zhaoji 1974@126.com
}

Received 4 September 2014; revised 4 October 2014; accepted 1 November 2014

Copyright (C) 2014 by authors and Scientific Research Publishing Inc.

This work is licensed under the Creative Commons Attribution International License (CC BY). http://creativecommons.org/licenses/by/4.0/

c) (i) Open Access

\begin{abstract}
Medical image segmentation is one of the key technologies in computer aided diagnosis. Due to the complexity and diversity of medical images, the wavelet multi-scale analysis is introduced into GVF (gradient vector flow) snake model. The modulus values of each scale and phase angle values are calculated using wavelet transform, and the local maximum points of modulus values, which are the contours of the object edges, are obtained along phase angle direction at each scale. Then, location of the edges of the object and segmentation is implemented by GVF snake model. The experiments on some medical images show that the improved algorithm has small amount of computation, fast convergence and good robustness to noise.
\end{abstract}

\section{Keywords}

Pattern Recognition, Image Segmentation, GVF Snake Model, Wavelet Multi-Scale Analysis, Medical Image

\section{Introduction}

Image segmentation is used widely in medical fields, such as medical research, clinical diagnosis and treatment, efficacy evaluation, image information processing, surgical planning, computer-assisted surgery, pathological analysis, image-guided surgery and surgical simulation [1]-[3]. In these applications, the most important and essential aspect is the image segmentation whose application is usually located in a bottleneck situation, consequently the work of medical image segmentation is very difficult, especially to complex image with much noise and the fuzzy boundary images.

At present, the common methods used in the perspective of medical applications include region-based me- 
thods, edge-based approach, combination of theoretical tool-specific methods (such as artificial neural networks and deformable model method), the methods based on statistical theory, fractal-based methods and methods based on mathematical morphology [4]. But the different medical image processing methods have different emphasis. As a result of the complexity and diversity of medical images, it is rather difficult to use only one of these methods to extract the edges correctly and accurately. So we should combine a number of ways to take advantage of different methods for learning from each other to make the medical image segmentation more accurate. Currently, there are some novel methods which are the combination of different methods, and the traditional methods are examined by assessing their advantages and disadvantages. The present paper proposes an improved mechanism in which deformable models that have been extensively researched and used in the field of medical image segmentation has far-reaching prospects. In this paper, the improved algorithm is based on the deformable model called gradient vector flow method combined with wavelet analysis.

\section{Background}

\subsection{GVF Deformable Model}

The earliest deformable model was presented in 1987 in the creative thesis "Snakes: Active Contour Models" of Kass [5]. It is applied to the object-segmentation and the video track detection and is a relatively good image analysis method. According to the theoretical background to solve problems, the Active Contour Models include two types of models. One is parametric deformable model [6]-[8], and the other is geometric deformable model [9]-[11]. Although the two methods are essentially different, their basic principle is similar [12]. In this paper, we focus on parametric deformable models, which synthesize parametric curves or surfaces within an image domain and allow them to move toward desired features, usually edges. Typically, the models are drawn toward the edges by potential forces, which are defined to be the negative gradient of potential functions.

In the parametric deformable models, the images segmentation process is to find the parameters that minimize the sum of internal and external energy which have been weighted. Usually, the minimum value is obtained in the image boundary which is the required image region. The energy minimization process is a joint decision of the internal forces and external forces. The internal forces include elastic force and bending force, whose primary role is to prevent excessive bending curve. The main role of the external force is to move the curve toward the boundaries of the image. They both make the curve gradually arrive at the image characteristics region which is the edges of the image. A traditional 2-D parametric deformable model or deformable contour is a curve $X(s)=(x(s), y(s), s \in[0,1])$ which moves through the spatial domain of an image to minimize the energy functional

$$
\varepsilon(X)=\int_{0}^{1} E_{\text {int }}(X(s))+E_{\text {ext }}(X(s)) \mathrm{d} s
$$

where $E_{\text {int }}$ is a scalar potential function. The external potential function $E_{\text {ext }}$ is derived from the image, so that it takes on its smaller values at the features of interest, such as boundaries. A deformable contour that minimizes must satisfy the Euler equation

$$
\alpha X^{\prime \prime}(s, t)-\beta X^{\prime \prime \prime}(s, t)-\nabla E_{\mathrm{ext}}=0
$$

To find a solution to Equation (2), the deformable contour is made dynamic by treating $x$ as function of time $t$ as well as $s$, i.e. $X(s, t)$. Then, the partial derivative of $x$ with respect to $t$ is set as follows:

$$
X_{t}(s, t)=\alpha X^{\prime \prime}(s, t)-\beta X^{\prime \prime \prime}(s, t)-\nabla E_{\text {ext }}
$$

In 1998, Xu proposed a new model, which is called gradient vector flow (GVF) field [13] [14]. This external force is computed as a diffusion of the gradient vectors of a gray-level or binary edge map derived from the image. The GVF deformable model's initializations can be inside, outside, or across the object's boundary. Unlike deformable models that use pressure forces, a GVF deformable model does not need prior knowledge about whether to shrink or expand toward the boundary, which is also one of the reasons that we choose GVF model as the segmentation algorithm.

Compared with traditional snake model, the GVF model has made significant improvements, and is widely used in medical image segmentation [15]-[18]. But the GVF model has certain requirements for the initial contour for the complex images. Therefore, further improvement is necessary, especially to improve the ability of 
capture range, which will help the process of convergence of the deformable model. For the fuzzy, noisy, dumbbell-shaped and other special images, the GVF convergence is more difficult. At the same time, calculating the entire image gradient vector flow needs a large amount of computation, which limits its application. Therefore, we propose a novel algorithm that the wavelet analysis theory is introduced into the GVF model to greatly improve the accuracy and speed of edge extraction.

\subsection{Wavelet Multi-Scale Edge Detection Principle}

Edge detection plays an important role in image analysis and computer vision. Edges characterize object boundary on the basis of the properties of intensity and texture, which can provide valuable information for further image processing such as image segmentation, image enhancement and image compression. In early years, many edge detection algorithms have been developed [19].

The application of wavelet transform in image processing has received significant attention and some very efficient wavelet-based multi-scale edge detection algorithms have been proposed [20]. This is due to the fact that wavelet transform can provide a multi-resolution representation for an image with flexible localization in both time and frequency domains [21] [22].

In 1992, Mallat and Zhong conducted some research on sudden changes in signals and their characteristics under wavelet transforms. They also studied the properties of multi-scale edges through the wavelet theory and showed that the evolution of wavelet, local maxima across scales would characterize the local shape of irregular structure.

The image often is the two-dimensional signal. Suppose $\theta(x, y)$ is a two-dimensional smoothing function, while $\iint_{R R} \theta(x, y) \mathrm{d} x \mathrm{~d} y=1$ and $\lim _{x, y \rightarrow \infty} \theta(x, y)=0$, and in the $X$ and $Y$ direction, the partial derivatives of twodimensional wavelet function are defined as follows:

$$
\Psi^{(1)}(x, y)=\frac{\partial \theta(x, y)}{\partial x}, \Psi^{(2)}(x, y)=\frac{\partial \theta(x, y)}{\partial y}
$$

Suppose $\theta(x, y)$ is a smoothing function, and $\Psi^{(1)}$ and $\Psi^{(2)}$ are the two derived wavelet functions. Suppose $s>0$ is a scaling factor, the wavelet transform of $f(x, y) \in L^{2}\left(R^{2}\right)$ at scale $s$ is a vector with two components $W_{s}^{1} f(x, y)$ and $W_{s}^{2} f(x, y)$, which are given as

$$
\begin{aligned}
& X \text { direction: } W_{s}^{1} f(x, y)=f * \psi_{s}^{1}(x, y) \\
& Y \text { direction: } W_{s}^{2} f(x, y)=f * \psi_{s}^{2}(x, y)
\end{aligned}
$$

The wavelet transform of $f(x, y)$ is that

$$
\left[\begin{array}{l}
W_{s}^{1} f(x, y) \\
W_{s}^{2} f(x, y)
\end{array}\right]=s\left[\begin{array}{l}
\frac{\partial}{\partial x}\left(f * \theta_{s}\right)(x, y) \\
\frac{\partial}{\partial y}\left(f * \theta_{s}\right)(x, y)
\end{array}\right]=s \nabla\left(f * \theta_{s}\right)(x, y)
$$

Since Dyadic Wavelet has "zoom" function on analysis of the signal, we only need to increase the magnification by reducing the value of $j$ if we want to watch the details of the signal. The wavelet transform of $f$ at scale $2^{j}$ is a vector with two components $W_{2^{j}}^{1} f(x, y)$ and $W_{2^{j}}^{2} f(x, y)$, which are defined as

$$
\begin{aligned}
& X \text { direction: } W_{2^{j}}^{1} f(x, y)=f * \psi_{2 j}^{1}(x, y), \\
& Y \text { direction: } W_{2^{j}}^{2} f(x, y)=f * \psi_{2 j}^{2}(x, y) .
\end{aligned}
$$

The Dyadic Wavelet transform of two-dimensional image is

$$
\left[\begin{array}{l}
W_{2^{j}}^{1} f(x, y) \\
W_{2^{j}}^{2} f(x, y)
\end{array}\right]=2^{j}\left[\begin{array}{l}
\frac{\partial}{\partial x}\left(f * \theta_{2^{j}}\right)(x, y) \\
\frac{\partial}{\partial y}\left(f * \theta_{2^{j}}\right)(x, y)
\end{array}\right]=2^{j} \nabla\left(f * \theta_{2^{j}}\right)(x, y)
$$


Respectively, the image gradient modulus $M_{2^{j}} f(x, y)$ and amplitude angle $A_{2^{j}} f(x, y)$ are as follows

$$
\begin{gathered}
M_{2^{j}} f(x, y)=\sqrt{\left|W_{2^{j}}^{1} f(x, y)\right|^{2}+\left|W_{2^{j}}^{2} f(x, y)\right|^{2}} \\
A_{2^{j}} f(x, y)=\operatorname{tg}^{-1} \frac{\left|W_{2^{j}}^{2} f(x, y)\right|}{\left|W_{2^{j}}^{1} f(x, y)\right|}
\end{gathered}
$$

Modulus value of wavelet transform is proportional to the gradient vector-valued. Wavelet transform amplitude angle $A_{2^{j}} f(x, y)$ is the angle between the gradient vector $W_{2^{j}} f(x, y)$ and the horizontal direction which is image edge direction. So, if we want to detect the edges, we only need to find the local maximum modulus points of the gradient vector [23] [24].

\subsection{Implementation of Wavelet Edge Detection}

Figure 1 shows several edge detection results using traditional edge detection operators and multi-scale wavelet transform method on medical image. Figure 1(a) is an original image which is a head MR image whose size is $256 \times 256$. Since the Gaussian function has a nice feature in the frequency domain and time domain, we select the Gaussian function $\theta(x, y)$ as a smoothing function which is a low-pass filtering operator. So the wavelet functions are partial derivative of 2-D Gaussian function. The results using the traditional edge detection operators such as Canny operator, Log operator, Prewitt operator, Sobel operator and the zero cross method are shown from Figure 1(b) to Figure 1(g). As shown in Figure 1(h), the wavelet transform method is applied to extract the edges of the image with scale $s=2$, threshold $=0.3$.

The comparison of the experimental results shows that the edges extracted using the traditional operator is very wide, more obscure, and has lost a lot of image details. And when the smoothness of the edge is poor, more non-linear noise is retained. However, the wavelet transform modulus maxima approach can accurately position the edges affected by noise and the edge is one pixel wide. On a large scale, the wavelet transform modulus maxima method obtains major edge information. On the small scale, it obtains the details of the edge information. This feature is suitable for us to identify and locate objects from coarse to fine. The experimental results show that wavelet transform can detect weak edge which is not detected by the other algorithms, and has good continuity and smoothness. In short, the image edge detection method based on wavelet transform has a good application in medical image.

Figure 2 shows the different effects of edge detection by wavelet transform modulus maxima algorithm with different threshold values and scaling factors. The experimental results show that the larger scaling factor is suitable for extraction of the main outline; on the contrary, the smaller scaling factor is suitable for the extraction

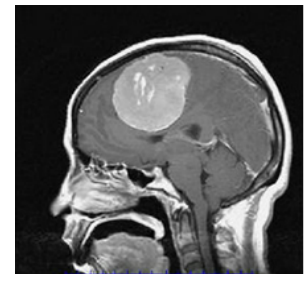

(a)

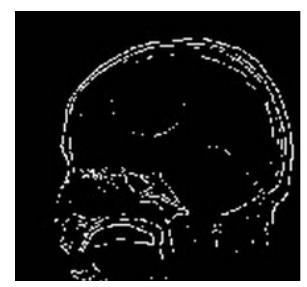

(e)

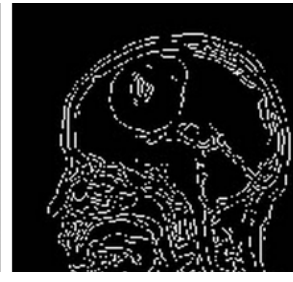

(b)

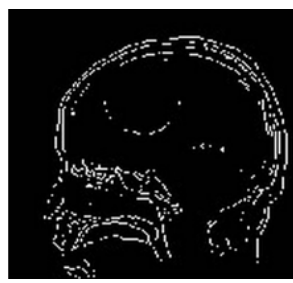

(f)

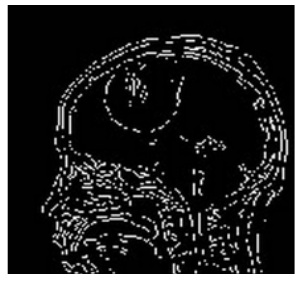

(c)

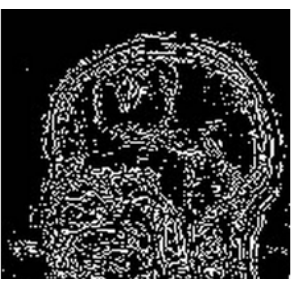

(g)

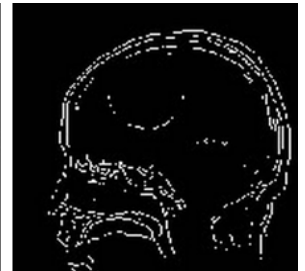

(d)

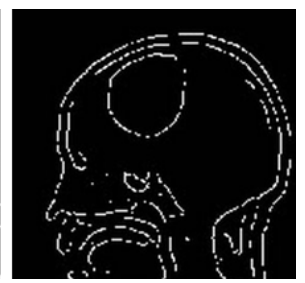

(h)

Figure 1. (a) Original image; (b) Canny operator; (c) Log operator; (d) Prewitt operator; (e) Robert operator; (f) Sobel operator; (g) Zero cross; (h) Wavelet method. 

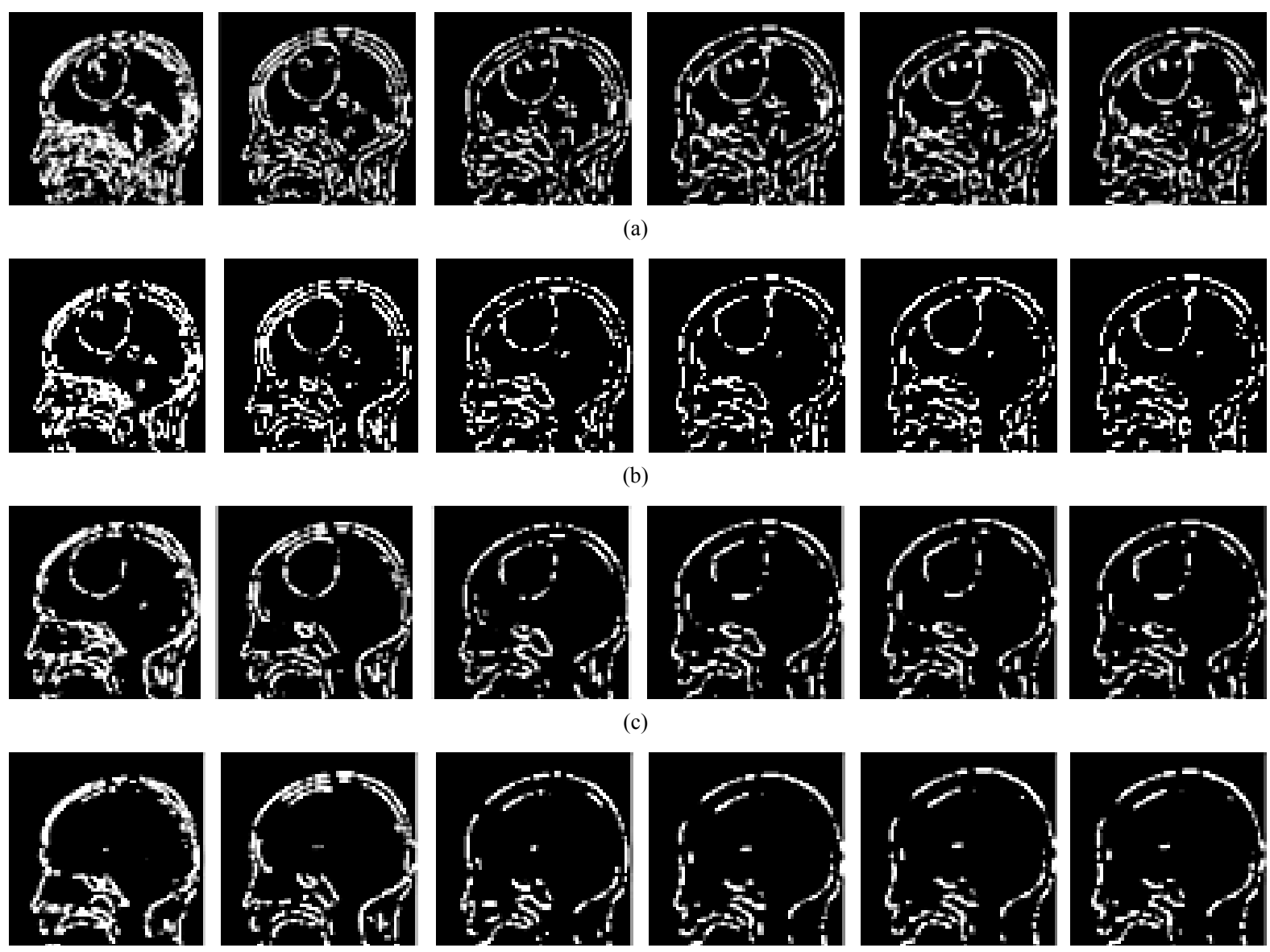

(d)

Figure 2. (a) Threshold $=0.1$; (b) Threshold $=0.2$; (c) Threshold $=0.3$; (d) Threshold $=0.4$. Scaling factor $=1,2,3,4,5,6$ from left to right.

of image details. The appropriate threshold can not only get rid of the pseudo-edge of the image, but also remove some noise, while too big threshold may filter out the useful edge information.

\section{Experimental Results and Analysis}

First, the edge points of the interested field are detected by using the wavelet transform, and then the Gradient Vector Flow field is constructed on the basis of edge detection results. Then the target segmentation is realized by using the GVF parameter deformable model. We adopt the wavelet transform and the GVF contour model combination algorithm and apply it to a variety of medical image object segmentation and obtain good results. In this paper, the experimental algorithm works on the environment of CPU: Celeron $1.1 \mathrm{GHz}$, Memory: $256 \mathrm{M}$, Operating System: Windows Xp and Simulation Software: Matlab 2010a.

In the experiment, we selected five different characteristic of the pictures as the experimental data whose size is $256 \times 256$. As indicated in Figure 3, Figure 3(a) is a high-resolution human brain skull picture by magnetic resonance; Figure 3(b) is an edge fuzzy chest CT picture; Figure 3(c) is a picture of a head with concavity by $\mathrm{CT}$ on ventricle level; Figure 3(d) is a head picture added noise by magnetic resonance at eye level, and Figure $3(e)$ is the homogeneous gray liver picture by magnetic resonance. Respectively, we will apply GVF method and our proposed combination algorithm with wavelet transform, and the GVF model whose parameters are $\alpha=0.05, \beta=0, \mu=0.2$ into the different medical image for segmentation.

First, we show several examples of the GVF field computations on medical images and demonstrate the limitation of key properties of the GVF deformable contours. The deformable contours were dynamically reparameterized to maintain contour point separation to within $0.5-1.5$ pixels. All edge maps used in GVF computations 
were normalized to the range $[0,1]$.

The GVF model has a much larger capture range and better concavity convergence on simple images. In our experiment, however, the results demonstrate that the GVF model has a poor performance on medical images as shown in Figure 4 and Figure 5. Figure 4 and Figure 5 show the initialization, the convergence of a deformable contour and the final results using GVF external forces. The initial contour position is inside the desirable region in Figure 4(c), and outside or across the desirable region in Figure 5(c) with same parameters. Clearly, the merits of the GVF deformable contour, which has a broad capture range and superior convergence, are not demonstrated. Another reason is that the GVF forces field of medical image is more complex than the simple image. The evolution of the GVF contour will deviate from the correct direction due to the interference of other force field, which is generated by noise or other objects of the image, and finally lead to segmentation failure.

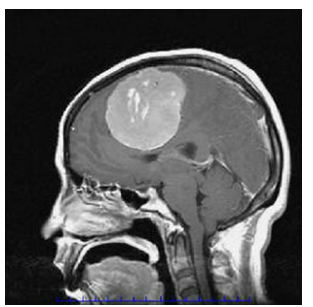

(a)

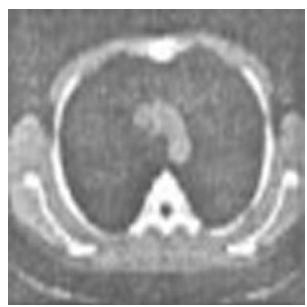

(b)

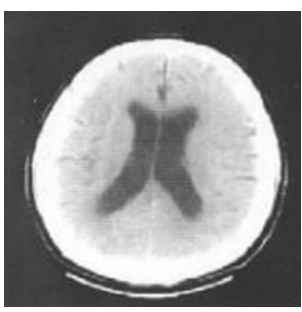

(c)

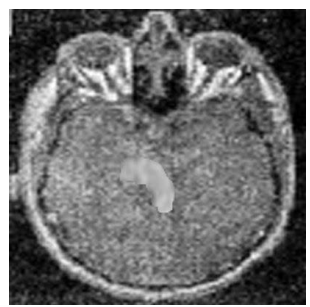

(d)

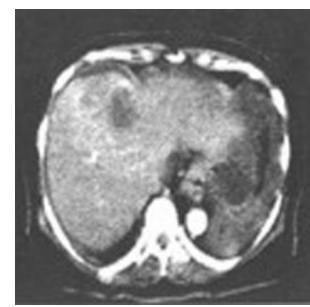

(e)

Figure 3. Five different medical images.
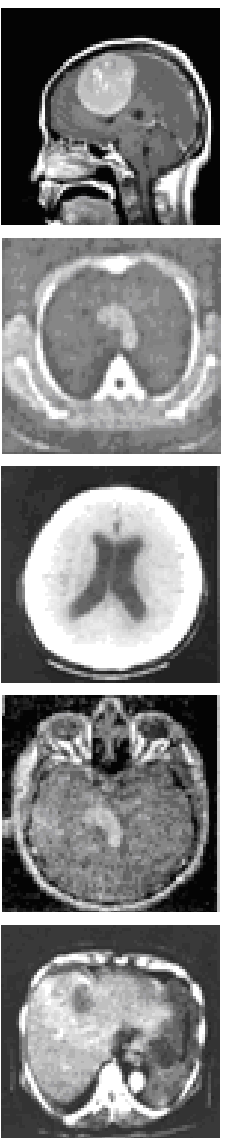

(a)
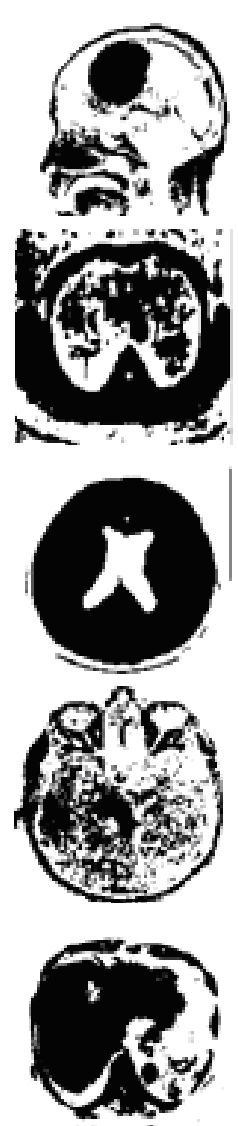

(b)
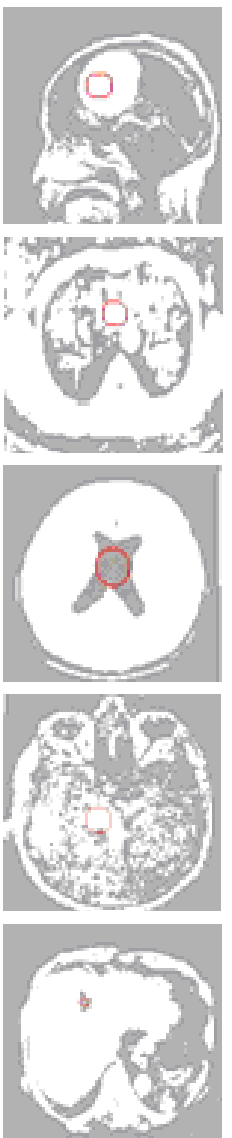

(c)
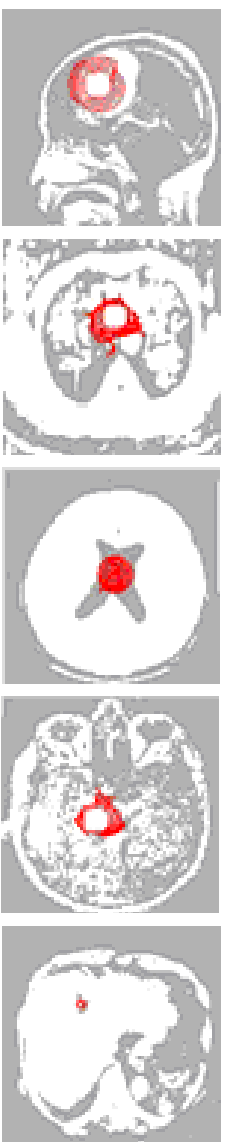

(d)
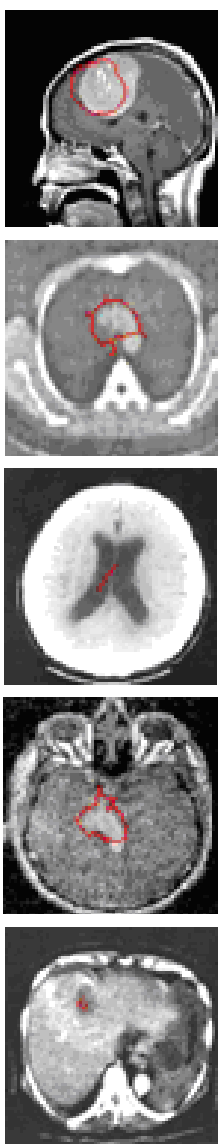

(e)

Figure 4. (a) Five different medical images; (b) Binaryzation map; (c) Initialization inside or across the interested region; (d) The convergence of a deformable contour using GVF external forces; (e) Final segmentation results by the GVF deformable contour model. 
In this paper, we introduce the wavelet modulus maxima method to improve the implementation of the GVF model. Figure 6(a) is the close-up of the edge gradient map with $\left|\nabla\left(G_{\sigma} * I\right)\right|^{2}$ for Figure 3(a). The close-up of gradient vector flow external force field of Figure 3(a) is shown in Figure 6(b). As we can see, the gradient vector flow field is confusing. In our proposed method, to compute the gradient vector flow for gray-level images, the edge-map must first be calculated by wavelet transform, which can remove noise and the undesirable details on different scale. Figure 6(c) shows the edge-map calculated by wavelet modulus maxima method, on the basis of which we then calculate the gradient vector flow in Figure 6(d). It is evident that the desirable gradient vector flow field is retained, while the undesirable that is smoothed out.
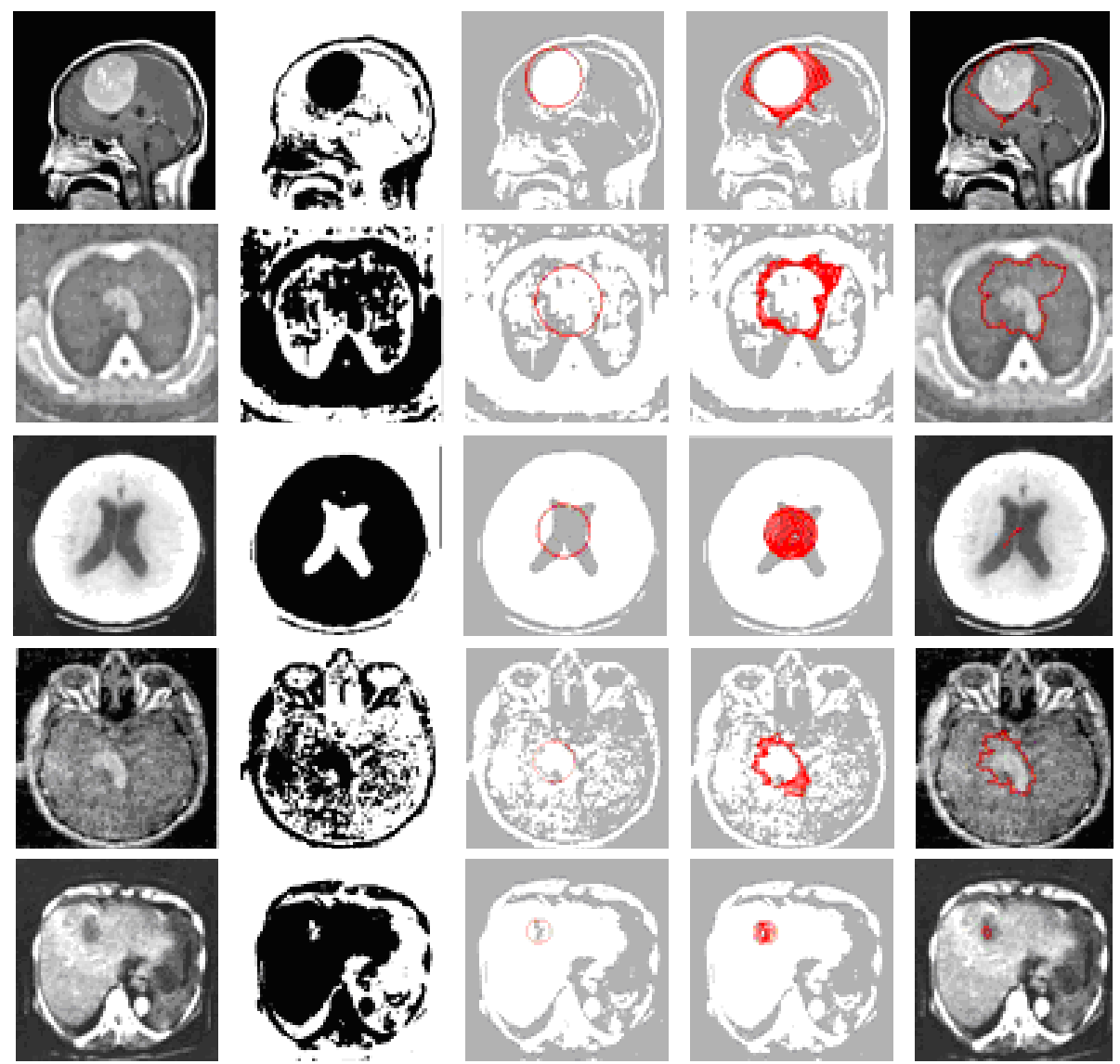

(a)

(b)

(c)

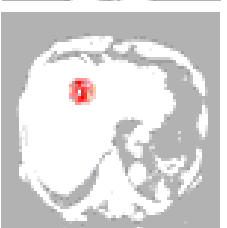

(d)

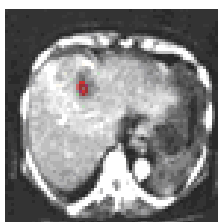

(e)

Figure 5. (a) Five different medical images; (b) Binaryzation map; (c) Initialization outside the interested region; (d) The convergence of a deformable contour using GVF external forces; (e) Final segmentation results by the GVF deformable contour model.

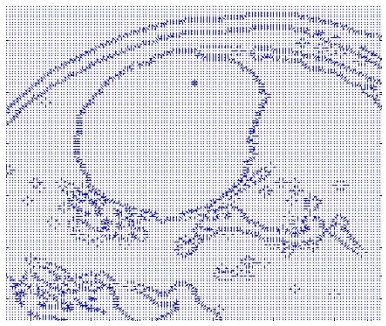

(a)

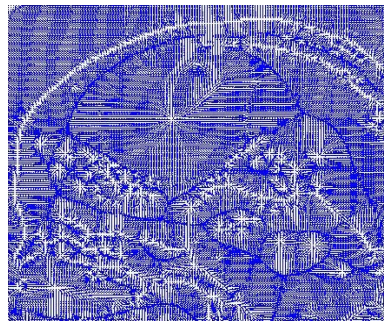

(b)

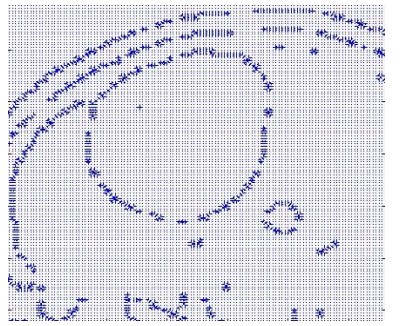

(c)

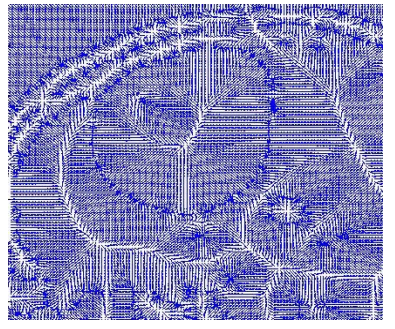

(d)

Figure 6. (a) Edge gradient map with $\left|\nabla\left(G_{\sigma} * I\right)\right|^{2}$; (b) Gradient vector flow external force field; (c) Wavelet transform modulus maxima edge map; (d) Gradient vector flow field on the basis of wavelet transform. 
Figure 7(a) and Figure 8(a) are the same as images segmented in Figure 4(a) and Figure 5(a). Figure 7(b) is the extracted edges by wavelet transform. Figure 7(c) is the edge maps of Figure 7(b). The identical initialization, initial contour position inside the interested region (Figure 7(d)) and outside or across the interested region (Figure 8(d)) is done. The configuration of convergence of the deformable contour using GVF external forces after the wavelet transform is shown in Figure 7(e) and Figure 8(e). Figure 7(f) and Figure 8(f) are the final segmentation results. As we can see, the results show that the new algorithm has an excellent convergence to the boundary, despite the far initialization, noise, and the boundary concavity. The comparison between traditional GVF model and the new algorithms on effectiveness of the implementation is shown in Table 1.

Respectively, Table 2 and Table 3 list the number of iterations and running time using the GVF model or the proposed algorithm on the five different characteristic of medical images. The experimental results show that the proposed algorithm can use fewer numbers of iterations and running time to achieve better segmentation results.

Figure 9 compares the running time using the GVF algorithm and the proposed algorithm. It can be seen from Figure 9 that the running time of the proposed algorithm is less than that of the GVF model under the same experimental environment and algorithm parameters. The reason is that the GVF model requires a lot of time to calculate the gradient vector flow field of the image domain. The new algorithm saves the running time by removing the noise and the undesirable details which might interfere with performance of the evolution curve.
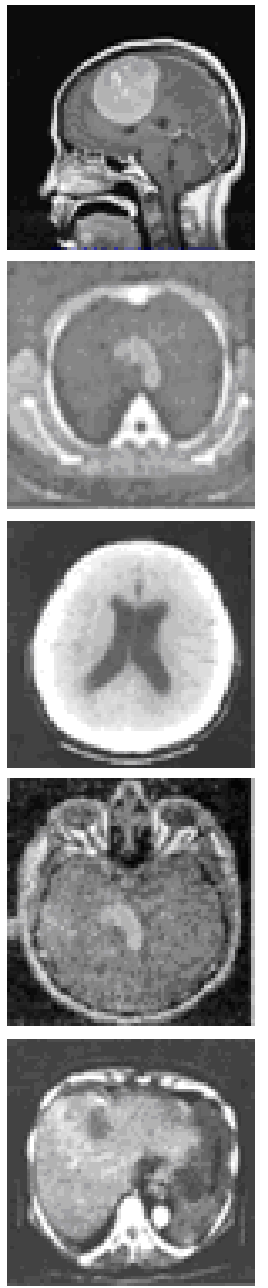

(a)
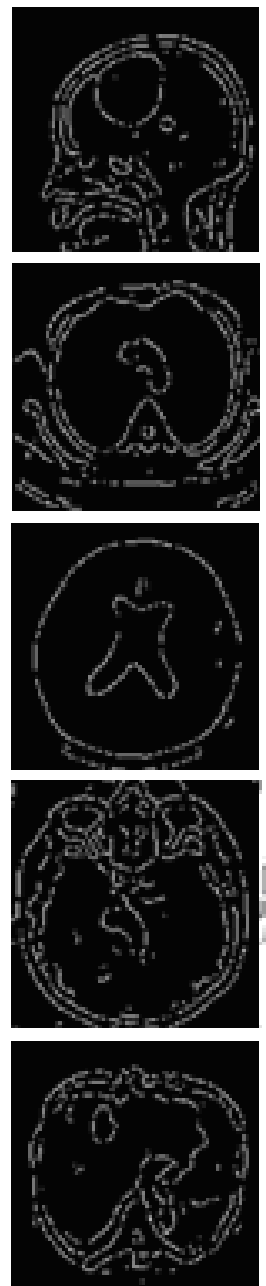

(b)
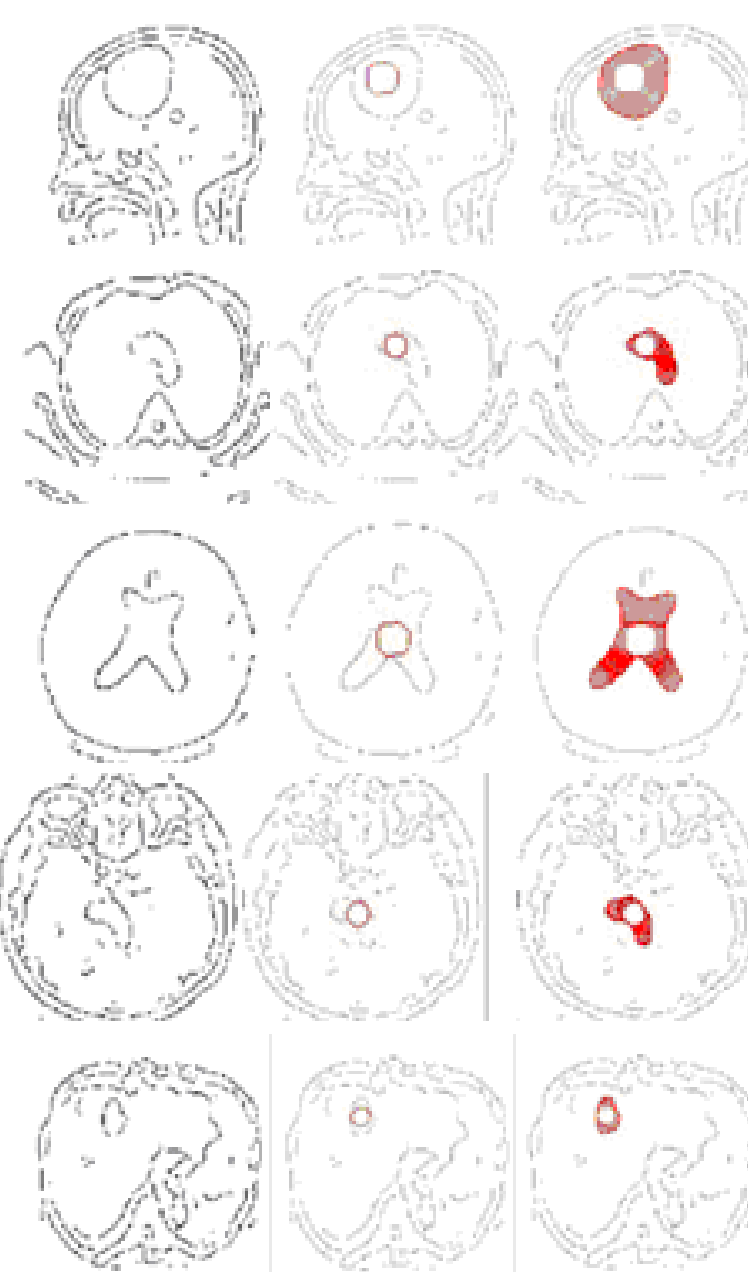

(c)
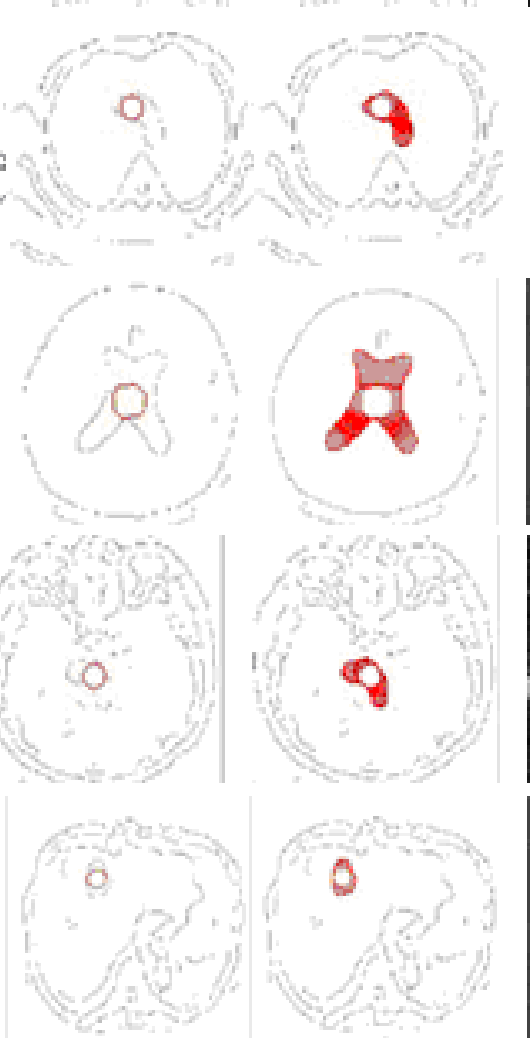

(d)
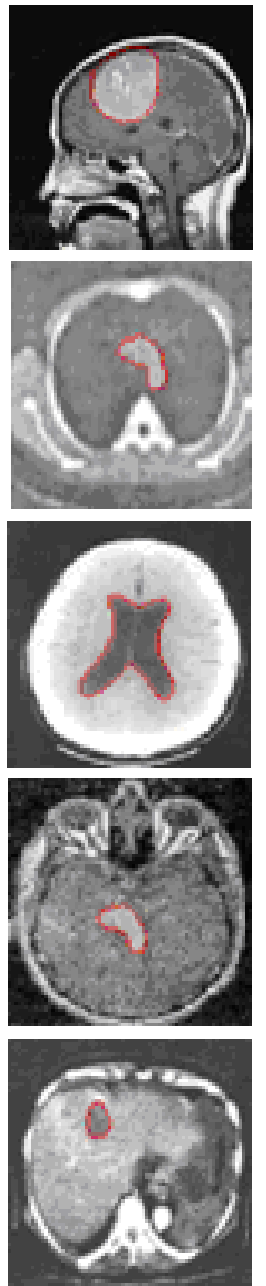

(f)

Figure 7. (a) Five different medical images; (b) Edge extraction by wavelet transform; (c) Edge map; (d) Initial contour position inside the interested region; (e) The convergence of a deformable contour using GVF external forces; (f) Final results of the GVF deformable contour. 

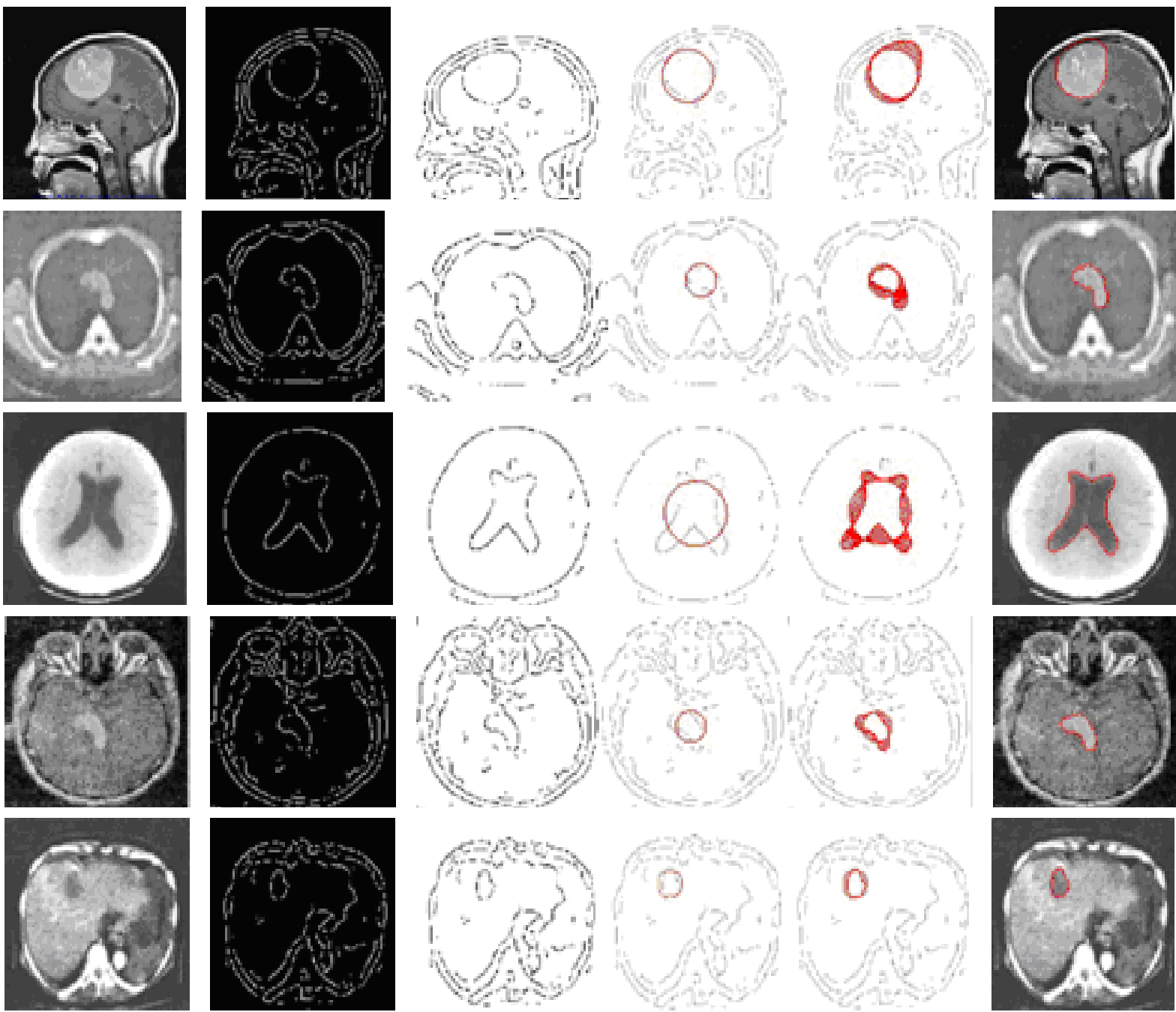

(a)

(b)

(c)

(d)

(f)

Figure 8. (a) Five different medical images; (b) Edge extraction by wavelet transform; (c) Edge map; (d) Initial contour position outside the interested region; (e) The convergence of a deformable contour using GVF external forces; (f) Final result of the GVF deformable contour.

Table 1. The comparison of algorithm performance.

\begin{tabular}{|c|c|c|c|c|c|}
\hline \multirow{4}{*}{ Image Quality } & \multirow{4}{*}{ Performance Analysis } & \multicolumn{2}{|c|}{ GVF Algorithm } & \multicolumn{2}{|c|}{ The Proposed Algorithm } \\
\hline & & \multicolumn{2}{|c|}{ Initial Contour } & \multicolumn{2}{|c|}{ Initial Contour } \\
\hline & & Inside & Outside or Across & Inside & Outside or Across \\
\hline & & \multicolumn{4}{|c|}{ Whether the interested region is accurately extracted? } \\
\hline Figure 4(a) & high-resolution & No & No & Yes & Yes \\
\hline Figure 4(b) & fuzzy & No & No & Yes & Yes \\
\hline Figure 4(c) & concavity & No & No & Yes & Yes \\
\hline Figure 4(d) & noisy & No & No & Yes & Yes \\
\hline Figure 4(e) & homogeneous gray & No & No & Yes & Yes \\
\hline
\end{tabular}


Table 2. Number of iterations and running time of the GVF algorithm.

\begin{tabular}{lcccc}
\hline & \multicolumn{3}{c}{ Initial Contour } \\
\cline { 2 - 5 } & \multicolumn{2}{c}{ Inside } & Outside or Across \\
\cline { 2 - 5 } & Number of Iterations & Running Time & Number of Iterations & Running Time \\
\hline Figure 4(a) & 60 & 15.2500 & 50 & 13.6406 \\
Figure 4(b) & 160 & 42.5938 & 130 & 36.2813 \\
Figure 4(c) & 250 & 81.1406 & 90 & 22.0313 \\
Figure 4(d) & 100 & 35.4219 & 30 & 13.0625 \\
Figure 4(e) & 30 & 14.8750 & 30 & 9.4844 \\
\hline
\end{tabular}

Table 3. Number of iterations and running time of the proposed algorithm.

\begin{tabular}{lcccc}
\hline & \multicolumn{3}{c}{ Initial Contour } \\
\cline { 2 - 5 } & \multicolumn{2}{c}{ Inside } & \multicolumn{2}{c}{ Outside or Across } \\
\cline { 2 - 5 } & Number of Iterations & Running Time & Number of Iterations & Running Time \\
\hline Figure 4(a) & 50 & 10.1094 & 30 & 6.3281 \\
Figure 4(b) & 150 & 27.7813 & 125 & 24.00 \\
Figure 4(c) & 240 & 48.9531 & 85 & 17.6094 \\
Figure 4(d) & 50 & 18.1875 & 20 & 5.5469 \\
Figure 4(e) & 15 & 6.4063 & 10 & 3.7656 \\
\hline
\end{tabular}

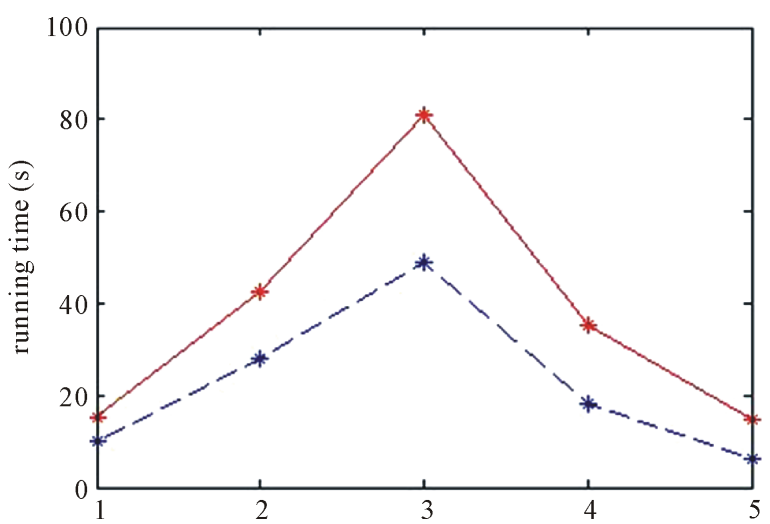

(a)

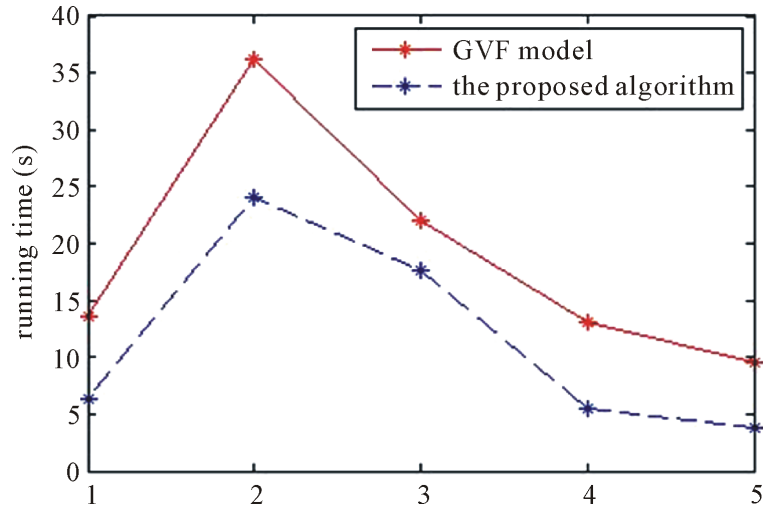

(b)

Figure 9. (a) Initial contour inside the interested region; (b) Initial contour outside or across the interested region.

In the future, some investigations into the features and uses of the improved GVF model would be warranted. It would also been done to understand the interplay between the GVF parameters and the wavelet transform parameters and select the parameters adaptively. Likewise, the novel algorithm framework might be useful in defining new improved parametric and geometric deformable models. Finally, making relationship between improved GVF model with other applications in image processing, computer vision, and medical imaging might provide some new insights or even new solutions to existing problems (Figure 9).

\section{Conclusion}

Related to the traditional parameters deformable model, the GVF deformable model has been greatly improved, 
but certain requirements of the initial contour position and more computation time are still needed. Since the wavelet transform has the features of edge detection and noise removal, in this paper, the wavelet transform modulus maxima is introduced into the GVF model to detect the edge of the image and remove the noise and the undesirable details. Experimental results show that the novel algorithm can use less running time to obtain better segmentation results. The dynamic behavior of the GVF model in the energy minimization process manifests that the new algorithm has a wide range of applications, especially in medical image analysis and disease diagnosis.

\section{Acknowledgements}

This Research is funded by the Education Department of Liaoning Province Foundation grant Number LJQ2014033 and University of Science and Technology Liaoning Foundation grant Number 2013RC08.

\section{References}

[1] Gao, J., Li, X.W. and Zhang, J. (2007) The Research of Image Preprocessing Algorithm in Color Print Quality Inspection System. Packaging Engineering, 28, 64-66.

[2] Feng, J.H. and Gong, J.L. (2010) Improved Gradient Vector Flow Model and Its Applications. Journal of Numerical Methods and Computer Applications, 31, 30-38.

[3] Li, K.C. and Lu, J. (2013) Progress in Medical Imaging. Journal of Capital Medical University, 34, $364-367$.

[4] Pham, D.L., Xu, C.Y. and Prince, J.L. (1998) A Survey of Current Method in Medical Image Segmentation. Technical Report JHU/ECE 99-01, Johns Hopkins University.

[5] Kass, M., Witkin, A. and Terzopoulos, D. (1987) Snakes: Active Contour Models. International Journal of Computer Vision, 1, 321-331,

[6] Cohen, L.D. and Cohen, I. (1993) Finite-Element Methods for Active Contour Models and Balloons for 2D and 3D Images. IEEE Transactions on Pattern Analysis and Machine Intelligence, 15, 1131-1147.

[7] McInerney, T. and Terzopoulos, D. (1995) A Dynamic Finite Element Surface Model for Segmentation and Tracking in Multidimensional Medical Images with Application to Cardiac 4D Image Analysis. Computerized Medical Imaging and Graphics, 19, 69-83.

[8] Terzopoulos, D. and Fleischer, K. (1988) Deformable Models. The Visual Computer, 4, 306-331.

[9] Caselles, V., Catte, F., Coll, T. and Dibos, F. (1993) A Geometric Model for Active Contours. Numerische Mathematik, 66, 1-31.

[10] Chan, T. and Vese, L.A. (2001) Active Contours without Edges. IEEE Transactions on Image Processing, 10, 266277.

[11] Li, C.M., Xu, C.Y., Gui, C.F. and Fox, M.D. (2005) Level Set Evolution without Re-Initialization: A New Variational Formulation. IEEE Computer Society Conference on Computer Vision and Pattern Recognition, 1, 430-436.

[12] Xu, C.Y., Yezzi, A. and Prince, J.L. (2000) On Relationship between Parametric and Geometric Active Contours. Proceedings of 34th Asilomar Conference on Signal, Systems and Computers, Pacific Grove, 29 October-1 November 2000, 483-489.

[13] Xu, C.Y. and Prince, J.L. (1998) Snakes, Shapes, and Gradient Vector Flow. IEEE Transactions on Image Processing, 7, 359-369. http://dx.doi.org/10.1109/83.661186

[14] Xu, C.Y. and Prince, J.L. (2000) Gradient Vector Flow Deformable Models. In: Handbook of Medical Imaging.

[15] Alomari, R.S., Kompalli, S. and Chaudhary, V. (2008) Segmentation of the Liver from Abdominal CT Using Markov Random Field Model and GVF Snakes. Proceedings-CISIS 2008: 2nd International Conference on Complex, Intelligent and Software Intensive Systems, Barcelona, 4-7 March 2008, 293-298.

[16] Wu, X.G., Spencer, S.A., Shen, S., Fiveash, J.B., Duan, J. and Brezovich, I.A. (2009) Development of an Accelerated GVF Semi-Automatic Contouring Algorithm for Radiotherapy Treatment Planning. Computers in Biology and Medicine, 39, 650-656. http://dx.doi.org/10.1016/j.compbiomed.2009.05.001

[17] Tao, G.Z., Singh, A. and Bidaut, L. (2010) Liver Segmentation from Registered Multiphase CT Data Sets with EM Clustering and GVF Level Set. Progress in Biomedical Optics and Imaging-Proceedings of SPIE, 7623. http://dx.doi.org/10.1117/12.844529

[18] Sen, X.B. (2011) Improved X-Ray Image Segmentation Based on GVF Snakes Model. Chinese Journal of Stereology and Image Analysis, 16, 1-4.

[19] Rafael, C.G. and Woods, R.E. (2004) Digital Image Processing. Publishing House of Electronics Industry, Beijing, 
460-482.

[20] Mallat, S. and Zhong, S. (1992) Characterization of Signals from Multiscale Edges. IEEE Transactions on Pattern Analysis and Machine Intelligence, 14, 710-732. http://dx.doi.org/10.1109/34.142909

[21] Mallat, S. and Hwang, W.L. (1992) Singularity Detection and Processing with Wavelets. IEEE Transactions on Information Theory, 38, 617-643. http://dx.doi.org/10.1109/18.119727

[22] Chen, T.L., Wang, J.F. and Zhang, K.Z. (2004) A Wavelet Transform Based Method for Road Centerline Extraction. Photogrammetric Engineering \& Remote Sensing, 70, 1423-1431. http://dx.doi.org/10.14358/PERS.70.12.1423

[23] Byeongseon, J. and Myungin, C. (2008) Construction of Symmetric Tight Wavelet Frames from Quasi Interpolatory Subdivision Masks and Their Applications. International Journal of Wavelets, Multiresolution and Information Processing, 6, 97-120. http://dx.doi.org/10.1142/S0219691308002240

[24] Niya, J.M. and Aghagolzadeh, A. (2004) Edge Detection Using Directional Wavelet Transform. IEEE Mediterranean Electrotechnical Conference, 1, 281-284. 
Scientific Research Publishing (SCIRP) is one of the largest Open Access journal publishers. It is currently publishing more than 200 open access, online, peer-reviewed journals covering a wide range of academic disciplines. SCIRP serves the worldwide academic communities and contributes to the progress and application of science with its publication.

Other selected journals from SCIRP are listed as below. Submit your manuscript to us via either submit@scirp.org or Online Submission Portal.
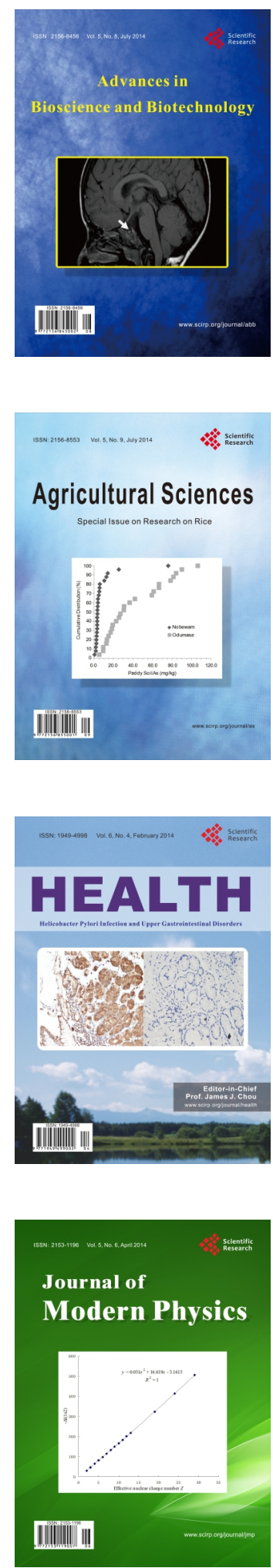
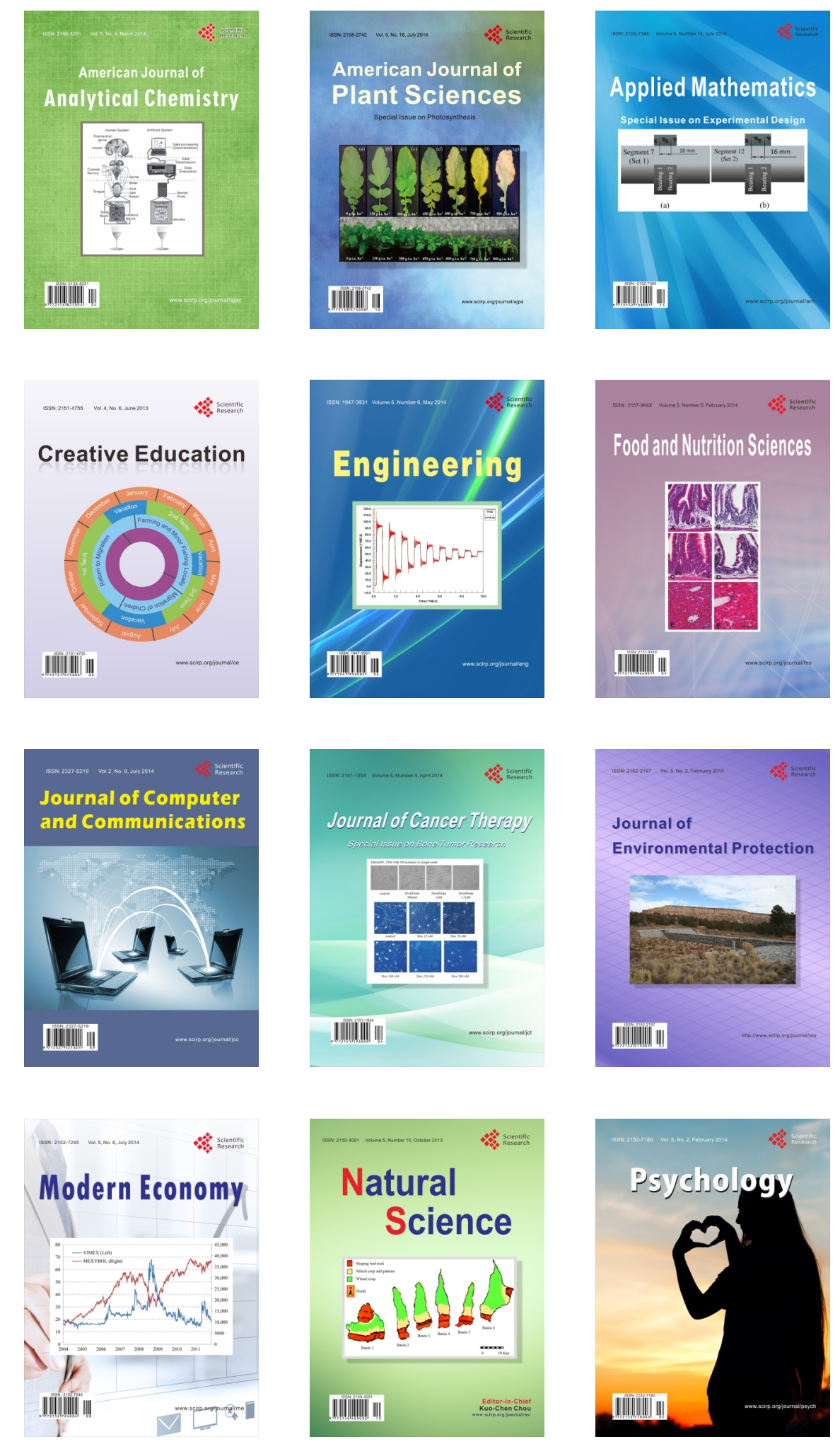